\title{
A Case of Pelvic Migraine
}

\author{
Conor O'Brien \\ Consultant Clinical Neurophysiologist, National Maternity Hospital, Dublin, Ireland \\ Email: drcob50@gmail.com
}

Received 26 August 2015; accepted 26 September 2015; published 29 September 2015

Copyright (C) 2015 by author and Scientific Research Publishing Inc.

This work is licensed under the Creative Commons Attribution International License (CC BY). http://creativecommons.org/licenses/by/4.0/

(c) (i) Open Access

\section{Abstract}

A 62-year-old woman presented with an 8-year history of chronic persisting pelvic pain. She described constant throbbing, stabbing vaginal pain. A pelvic floor neurophysiological assessment of the pudendal nerve was performed by performing a needle EMG to the left and right external anal sphincter assessing for insertional activity and recruitment pattern. A quantitative assessment of the motor unit action potentials [MUAPs] was also performed. Tests confirmed a left pudendal neuropathy with chronic denervation in the left external anal sphincter, with reasonable muscle function, with a recruitment pattern of $65 \%-70 \%$ of normal. The CAR showed an elevated sensory threshold with a normal distal latency. All other conventional pudendal nerve treatments including oral antiepileptic medication, neuromodulation and pudendal nerve blocking injections had failed, and the patient was exacerbated by the persisting pain and discomfort. In this case, 30 international units (iu) of botulinum toxin type $A$ in 10 divided doses of 3 iu were injected along the nerve. Four days later the patient reported a significant improvement in the pain symptoms. She was reviewed 3 weeks later and for the first time in 8 years had made the $\mathbf{7 0}$ mile journey to the clinic as a passenger in her husband's car. This case highlights a new therapeutic option of botulinum toxin type $A$ injection, along the nerve length, for this common painful condition. It seems to have clinical veracity as unlike other therapeutic option the affect lasts for 3 or 4 months.

\section{Keywords}

Pudendal Neuropathy, Vaginal Birth, Pelvic Pain, Migraine, Pelvic Migraine, Botulinum Toxin Injection, Pelvic Floor Neurophysiology

\section{Introduction}

Pudendal nerve dysfunction can occur in up to 1/3 of all vaginal births. It can result in faecal and urinary incontinence, as well as sexual dysfunction and pain. The pain in many cases presents as a throbbing discomfort, with sensory alteration in the perineum, which can be exacerbated by defecation or prolonged sitting. The throbbing discomfort mimics the headache associated with migraine. Pelvic pain is often described as "a headache in the 
pelvis”. The treatment of pelvic floor pain usually concentrates on relieving muscle spasm with myofascial release treatments being a popular therapy. Over the past decade, migraine headache therapy has included botulinum type A therapy at 39 points over the facial nerves. This has proven to have a positive effect on throbbing headache prevention. We presented a case of painful pudendal neuropathy which was successfully treated with a 10 points injection therapy of botulinum toxin A along the course of the pudendal nerve, in a similar methodology to the migraine therapy suggesting that the neuropathic pain was associated with pudendal neuropathy mirrors migraine headaches, and perhaps the condition could be renamed "pelvic migraine".

\section{Case Report}

\subsection{History}

A 62-year-old woman presented with an 8-year history of chronic persisting pelvic pain. She described constant throbbing, stabbing vaginal pain which she associated with episodes of burning. She also described constant perineal sensory alteration, with vulvar tingling, which she described a being like a "nettle sting" and numbness in the vaginal vault. The pain was exacerbated by defecation and symptoms subsided on urination. By the time of presentation, she was unable to travel in a motor car for long periods due to pain on prolonged sitting. She travelled in the main by bus as she could stand during the journey. Her problems started after a hysterectomy which was performed for uterine fibroids. She had no evidence of interstitial cystitis. She had 2 adult children, both delivered vaginally without complication. She had previously suffered from polio and developed a postpolio syndrome in her 30's which affected her right arm. She was referred for assessment of the pudendal nerve.

\subsection{Electrodiagnostic Assessment}

A pelvic floor neurophysiological assessment of the pudendal nerve was performed by performing a needle EMG to the left and right external anal sphincter assessing for insertional activity and recruitment pattern. A quantitative assessment of the motor unit action potentials [MUAPs] was also performed. The integral structure of the full length of the pudendal nerve was achieved by performing the Clitoral Anal Sacral Reflex (CAR) on the right and left sides. These 2 tests are recommended by the World Health Organisation (WHO) as the most appropriate neurophysiologic tests of pudendal nerve function and give clear clinical information of nerve function and the presence or absence of recovery [1] which will affect therapeutic options.

The electrodiagnostic assessment confirmed a left pudendal neuropathy with chronic denervation in the left external anal sphincter, with reasonable muscle function, with a recruitment pattern of $65 \%-70 \%$ of normal. The CAR showed an elevated sensory threshold with a normal distal latency.

\subsection{Treatment}

The patient was initially treated with a combination of oral medications which included anti-epileptic medication, anti-inflammatory medication which included both steroidal and non-steroidal agents, as well as oral sidianafil to improve pelvic blood supply. There was an initial improvement in the intensity of the pelvic pain. A 12 treatment course of posterior tibial nerve neuromodulation was performed over a 4-week period. Symptoms improved during the course of the therapy but symptoms regressed 2 weeks after the last treatment. Pudendal nerve blocking injections were previously performed with little affect. These were repeated with a similar negative outcome.

As in many cases of pudendal neuropathy the diagnosis was confirmed but the therapeutic options were limited. It was decided to administer a trial injection of botulinum toxin. This therapy had previously been successful in a case of spasmodic levator ani syndrome [2], and is often used in cases of resistant pelvic floor pain. Botulinum therapy has become a mainstay of migraine headache pain over the past number of years [3], with significant clinical outcomes using a 39 points injection montage over specific facial nerves and muscles [4]. The mechanism of action is not completely understood but it is thought to be due to a reduction in muscle spasm and a direct effect on sensory nerve function.

In this case 30 international units (iu) of botulinum toxin type A in 10 divided doses of 3 iu were injected along the nerve. The injections were guided using a combination of ultrasound guidance and nerve stimulation guidance injecting through a Teca MyoJect Luer Lock disposable hypodermic needle electrode and recording the stimulated response at the external anal sphincter. The initial injection was placed at the junction of the sac- 
rospinous and sacrotuberous ligament with injections being placed at $2 \mathrm{~cm}$ distances along the nerve length with the final 3 injections being placed in the perineum $2 \mathrm{~cm}$ lateral to the anus and vagina.

4 days later the patient reported a significant improvement in the pain symptoms. She was reviewed 3 weeks later and for the first time in 8 years had made the 70 miles journey as a passenger in her husband's car. She described herself as being pain free. At 3 months she described some mild symptoms on defecation. She had no symptoms of faecal or urinary incontinence. She had a repeat therapy at 4 months using a similar protocol and has remained symptom free and is capable of sitting for periods of hours.

\section{Discussion}

Pudendal neuropathy is a common but frequently unspoken cause of severe pelvic floor pain, sexual dysfunction as well as faecal and urinary incontinence [5]. It is also a cause of significant social isolation. The nerve can be injured during child birth and research has indicated that $1 / 3$ of all vaginal deliveries can result in a pudendal nerve injury with $1 / 3$ of these persisting with clinical dysfunction [6]. On this basis there are upwards of 1 million new cases of pudendal neuropathy every year in the USA. Up to the 1980's little was known about the condition. Patients frequently failed to highlight the problem associated with this nerve injury due to a combination of embarrassment and shame due to the intimate area that is affected by the nerve's dysfunction. Many patients simply suffer on without ever seeking help for this painful and disabling condition.

The nerve is formed by the $2^{\text {nd }}, 3^{\text {rd }}$ and $4^{\text {th }}$ sacral nerve roots and travels into the pelvis through the greater sciatic and lesser sciatic foramen before it travels through Alcock's Fashia. It gives off 3 terminal branches; the haemorrhoidal, perineal and clitoral [7]. The nerve can be injured at any point along its course but is frequently compromised by the baby's head at the level of the ischial spine during the $2^{\text {nd }}$ stage of labour. The nerve is also vulnerable during cycling by pressure on the saddle on the sacrotuberous and sacrospinous ligaments and is referred to as "Cyclist Syndrome" [8].

The majority of pelvic floor pain symptoms are a combination of neuropathic pain and muscle spasm. In this case the symptoms were predominantly neuropathic in nature, with burning pain and sensory alteration being the cardinal symptoms. The electrodiagnostic evaluation confirmed the neuropathy. The condition has also been referred to as "a head ache in the pelvis" and treatment protocols to reduce pelvic muscle spasm have been designed. The Stanford Protocol and the Wise-Anderson Protocol are regularly utilised by clinicians and are outlined in the publication "A Headache in the Pelvis" [9].

The myofascial release techniques and trigger point therapy assist in reducing muscle spasm but often fail in cases of pudendal neuropathy where symptoms return quickly due to the persisting pathology which triggers the spasm and pain.

Conventional therapy for the neuropathic type pain that occurs in cases of pudendal neuropathy usually includes a combination of [1] oral medication such as Gabapentin Pregablin, the anti-depressant Amitriptyline [2], neuromodulation both remotely at the Tbial nerve and directly at the sacral plexus, and [3] nerve blocking injection. All therapeutic modalities had failed and the patient was quite distressed by the constant pain, which had taken a significant toll on her quality of life. She was willing to try any non-conventional therapy to resolve her ongoing complaint.

The procedure involves injecting over the pudendal nerve. This carries the risk of injury to the pudendal artery which travels beside the nerve for much of its course. Therefore the potential risks of the procedure have to be carefully explained to the patient, and signed informed consent is essential. The procedure is made significantly safer by the use of ultrasound to identify the structures, and the use of the Doppler ultrasound modality to confirm the location of the vascular structures. Nerve stimulation of the pudendal nerve with External Anal Sphincter recording further improves the accuracy of the placement of the injection. The potential pitfalls of the procedure include disruption of the vascular structures, but careful needle placement using the 2 guidance methods significantly reduces this risk. Overdosing with the toxin will weaken muscles. This may have an effect on continence. However the use of low dose botulinum toxin at different sites, in a similar way to migraine therapy appears to get over this potential side effect. The procedure in this case was atraumatic and without complication. The only discomfort that the patient experienced was on the initial stimulation of the nerve by the needle electrode. This did not reoccur in the subsequent stimulations.

The advantage of this therapy in cases of resistant painful pudendal neuropathy is the relatively rapid onset of action, and the duration of therapeutic response. As with migraine repeat therapy may be required but only at 3 - 
4 months duration [4]. Hence the therapy has a significant and positive affect on quality of life, as the patient may have to attend 3 or 4 times a year, rather than the more frequent attendances required for myofascial therapy. The therapy also avoids the side effects associated with the oral medication used to treat neuropathic pain syndromes. The patient's view was that the further therapy, when necessary, was a small price to pay and considered the time input to be analogous to attending her hair dresser.

\section{Conclusion}

This case highlights a new therapeutic option of botulinum toxin type A injection, along the nerve length, for this common painful condition. It seems to have clinical veracity as unlike other therapeutic option the affect lasts for 3 or 4 months. Further research is required to quantify the therapeutic response and to clarify the affect that botulinum toxin has on the sensory function of peripheral nerves. However, in this case, the clinical outcome was rapid and sustained over a 3 months period-the methodology of the injection therapy being similar to that of migraine. The frequently encountered symptom of throbbing pelvic pain identified in this case of pudendal neuropathy, mirrors the throbbing headache found in over half of all migraine cases. Perhaps rather than a headache in the pelvis, should this type of pudendal nerve pain be retitled "Pelvic Migraine"?

\section{References}

[1] O’Brien, C., O’Herlihy, C. and O’Connell, P.R. (2004) Pudendal Neuropathy Is Best Determined by Full Neurophysiologic Assessment. American Journal of Obstetrics \& Gynecology, 191, 1836. http://dx.doi.org/10.1016/j.ajog.2004.05.095

[2] O’Brien, C. (2012) Levator Ani Syndrome-British Society of Clinical Neurophysiology. Joint Meeting with the Italian Society of Neurophysiology, Queens Square, September 2012.

[3] Berhman, R.A., Tucker, T. and Guyuron, B. (2003) Single Site Botulinum Toxin Type-A Injection for Elimination of Migraine Trigger Points. Headache, 43, 1085-1089. http://dx.doi.org/10.1046/j.1526-4610.2003.03210.x

[4] Schaefer, S.M., Gottschalk, C.H. and Jabbari, B. (2015) Treatment of Chronic Migraine with Focus on Botulinum Neurotoxins. Toxins, 7, 2615-2628. http://dx.doi.org/10.3390/toxins

[5] Benson, J.T. and Griffis, K. (2005) Pudendal Neuralgia, a Severe Pain Syndrome. American Journal of Obstetrics \& Gynecology, 192, 1663-1668.

[6] Fitzpatrick, M., O’Brien, C., O’Connell, P.R. and O’Herlihy, C. (2003) Patterns of Abnormal Pudendal Nerve Conduction Associated with Postpartum Faecal Incontinence. American Journal of Obstetrics \& Gynecology, 189, 730-735. http://dx.doi.org/10.1067/S0002-9378(03)00817-2

[7] Hruby, S., Ebmer, J. and Dellon, L. (2005) Anatomy of Pudendal Nerve at Urogenital Diaphragm-New Critical Site for Nerve Entrapment. Urology, 66, 9949-9952. http://dx.doi.org/10.1016/j.urology.2005.05.032

[8] Asplund, C. and Brkdul, T. (2007) Weiss BDI Genitourinary Problems in Bicyclists. Current Sports Medicine Reports, 6, 333-339.

[9] Wise, D. and Anderson, R.U. (2014) A Headache in the Pelvis. 6th Edition, Cataloging-in Publication Data, USA. 